\title{
CXCR4 Accelerates Osteoclastogenesis Induced by Non-Small Cell Lung Carcinoma Cells Through Self-Potentiation and VCAM1 Secretion
}

\author{
Tongquan Liao Wugui Chen Jing Sun Ying Zhang $\mathrm{Xu} \mathrm{Hu}$ \\ Sizhen Yang Hao Qiu Songtao Li Tongwei Chu \\ Department of Orthopedics, Xinqiao Hospital, Third Military Medical University (Army Medical \\ University), Chongqing, China
}

\section{Key Words}

CXCR 4 - Bone metastasis • Non-small cell lung carcinoma • Vascular cell adhesion molecule $1 \cdot$ ADAM 17

\begin{abstract}
Background/Aims: Non-small cell lung carcinoma (NSCLC) metastasis to bone leads to skeletal-related events and a poor quality of life. Unravelling the mechanism of metastasis is crucial for improving survival. Previous work has implicated the role of CXCR4 in bone metastasis; however, the underlying mechanisms are unknown. Methods: The human bone metastasis tissue samples were obtained from lung cancer patients during surgery with consents. The patients were followed up and the overall survival curve was analysed. The expression of CXCR4, VCAM1, and ADAM17 was measured with real-time PCR, western blot and immunochemistry staining in human tissue or NSCLC cell lines. The effects of CXCR4, soluble VCAM 1 and ADAM17 on NSCLC proliferation, migration and invasion were measured with CCK-8, monolayer scratch assay and transwell chamber, respectively. The amount of soluble VCAM1 in the conditioned medium was detected with ELISA. Tartrate-resistant acid phosphatasethe (TRAP) staining was performed to stain the multinucleated cells regarded as osteoclasts. Results: In this study, CXCR4 was found to be highly expressed in bone destruction area of metastatic NSCLC samples and related to poor survival in NSCLC patients with bone metastasis. CXCR4 potentiated NSCLC with enhanced proliferation and invasion abilities, while CXCR4 knockdown significantly suppressed the growth and invasion. Furthermore, CXCR4 promoted lung cancer-induced osteoclast differentiation with increased osteoclast formation. We also found that soluble VCAM1 (sVCAM1) secreted in NSCLC contributed to the osteoclastogenesis induced by CXCR4. The overexpression of CXCR4 increased sVCAM1, and the sVCAM1 secreted from CXCR4-overexpressing NSCLC cells recruited and arrested additional osteoclast progenitors to promote osteoclastogenesis. ADAM17 was confirmed to act as a

\begin{tabular}{ll}
\hline Tongwei Chu & Department of Orthopedics, Xinqiao Hospital, Third Military Medical University \\
& (Army Medical University) 183 Xinqiaozhengjie St, Shapingba District, Chongqing, 400037 (China) \\
& Tel. +86 13708388336, E-Mail chtw@sina.com
\end{tabular}
\end{abstract}


downstream mediator of CXCR4. The chemical inhibition of ADAM17 with TAPI-2 decreased sVCAM1 secretion and the number of TRAP+ osteoclasts. Conclusion: Taken together, these results indicated that CXCR4 potentiated NSCLC and promoted osteoclastogenesis through sVCAM1, which was cleaved by ADAM17. These data support the pivotal role of the cross talk between CXCR4 and ADAM17-VCAM1 in NSCLC-induced bone metastasis.

(C) 2018 The Author(s)

Published by S. Karger AG, Basel

\section{Introduction}

Lung cancer is the leading malignancy worldwide in men and the second-leading malignancy in women $[1,2]$. Primary lung cancer includes small cell lung carcinoma (SCLC) and non-small cell lung carcinoma (NSCLC), and the latter accounts for $85 \%$ of all lung cancer cases [3]. NSCLC frequently metastasizes to bone, which is often associated with skeletalrelated events (SREs), defined as pathologic fracture, spinal cord compression, bone pain and hypercalcaemia [4]. However, the underlying mechanisms of the metastatic capacity of NSCLC remain elusive.

CXCR4, a chemokine receptor, and its sole agonist CXCL12 have been reported to play an essential role in multiple solid tumours, including prostate [5], brain, breast [6], colon [7] and lung cancers $[8,9]$. There is growing evidence suggesting that the CXCL12/CXCR4 axis is important for tumour-related mechanisms such as chemotaxis, invasion, tumorigenicity, angiogenesis and proliferation. Higher levels of CXCR4 are associated with poor prognosis and a high risk of SRE development, which inversely correlates with quality of life and survival time in affected patients $[10,11]$. Metastasis to the bone and the consequent SREs processes through metastatic quiescence, formation of tumour cell niches and, finally, tumour cell growth. During cancer-induced bone destruction, extensive studies have demonstrated that osteoclast differentiation is a key process in cancer-associated bone resorption. CXCL12/ CXCR4 contributes to certain tumour-mediated metastasis [12, 13]; however, the role and mechanism of CXCR4 in the osteoclastogenesis of NSCLC metastasis to the bone remains largely unknown.

Metastatic bone lesions arise due to the intricate interaction between tumour cells and the bone microenvironment [14]. Tumour cells, osteoblasts, osteoclasts, bone extracellular matrix, the bone microenvironment and the niche are major components of a vicious cycle necessary for the initiation and development of metastatic lesions in the skeleton. The osteoclastogenic factors produced by localized tumour cells converge to increase the expression of the receptor activator of NF- $\mathrm{BB}$ ligand (RNAKL). RANKL then binds to the RANK receptor on osteoclast precursors and mediates osteoclastogenesis. Upon activation of RANKL and its receptor, CXCL12/CXCR4 efficiently potentiates the formation of osteoclasts and resorption by RAW264.7 cells [15]. Tumour-released osteoclastogenic factors that are regulated by CXCR4 may be of great value as therapeutic targets.

VCAM1 belongs to a transmembrane immunoglobulin family and is mainly expressed in stimulated endothelial cells [16]. VCAM1 can be catalysed to soluble VCAM1 (sVCAM1). The major receptor of VCAM1 is $\alpha 4 \beta 1$ (very late antigen-4, VLA-4), which is expressed in many haematopoietic cells, including lymphocytes, monocytes and granulocytes. In breast cancer, VCAM1 was shown to be involved in the micro-environmental interaction between tumour cells and stromal cells and facilitate the metastasis of breast cancer cells to the bone [17]. Additionally, the serum VCAM1 levels in NSCLC patients are significantly higher compared with those in healthy subjects. However, whether CXCR4 regulates VCAM1 during the bone metastasis of NSCLC remains unknown.

Soluble forms of adhesion molecules such as VCAM1 are thought to be a result of proteolytic cleavage. The disinterring metalloproteinases (ADAMs) have been shown to be involved in the majority of protein shedding. Previous studies have shown that ADAM17 is responsible for the shedding of numerous receptors for oncogenic growth factors, cytokines and adhesion molecules, including VCAM1 [18]. Meanwhile, aberrant ADAM17 expression is related to aggressive lung cancer and poor prognosis [19]. Recently, it was reported that 
ADAM17 could be activated by CXCL12-CXCR4 signalling in colon cancers [20]. Given the shedding power and the cross talk between CXCR4 signalling and ADAM17, we reasoned that CXCR4 and ADAM17 signalling might converge in the bone metastasis of NSCLC.

In this study, we detected the effects of CXCR4 on NSCLC potentiation, including the tumour cell migration, invasion and proliferation capability. Tumour-induced osteoclastogenesis was also evaluated. Herein, we found that CXCR4 potentiated the NSCLC capability of proliferation and invasion. Further, during the interaction between NSCLC and stromal cells, NSCLC promoted osteoclast activation through sVCMA1 secretion. The CXCL12/CXCR4 axis facilitated VCAM1 secretion by regulating the protease ADAM17, which cleaved the endogenous VCAM1.

\section{Materials and Methods}

\section{Reagents}

RANKL and TAPI-2 were purchased from R\&D Systems (Minneapolis, MN, USA). Recombinant human CXCL12 and AMD3100 were supplied by PeproTech (Rocky Hill, NJ, USA). Rabbit anti-CXCR4, rabbit antiADAM17 and rabbit anti-VCAM1 were obtained from Abcam (Cambridge, UK). Rabbit anti-GAPDH antibody was obtained from Beyotime Biotechnology (Jiangsu, China).

Tissue samples and follow-up

All 32 bone metastasis tissue samples were obtained from lung adenocarcinoma patients at the Department of Orthopaedics, Xinqiao Hospital, Third Military Medical University (Army Medical University). The tissue samples were fixed in 10\% neutral-buffered formalin for immunohistochemistry and TRAP staining studies. Thirty two human NSCLC tissue samples were obtained from patients who underwent surgical treatment between 2014 and 2015 at the Department of Orthopaedics, Xinqiao Hospital, Third Military Medical University (Army Medical University), Chongqing, China. All patients were followed up until October 2017. Overall survival was defined as the interval between the dates of surgery and death. The study was approved by the Ethics Committee of Third Military Medical University (Army Medical University).

\section{Cell culture}

The NSCLC cell lines A549, H1299 and H1975 were procured from the China Centre for Type Culture Collection (CCTCC, Wuhan, China). A549 was maintained in high-glucose DMEM (Gibco, Shanghai, China) supplemented with 10\% FBS (Gibco, Life Technologies, USA). H1299 and H1975 cells were cultured in RPMI 1640 (Gibco, Shanghai, China) containing $10 \%$ FBS. The cells were incubated at $37^{\circ} \mathrm{C}$ in $5 \% \mathrm{CO}_{2}$.

\section{Small interfering RNA (siRNA) transfection}

The CXCR4 siRNA or non-targeting control siRNA were synthesized by GenePharma (Shanghai, China). H1299 cells at 70-80\% confluence were transfected with siRNAs using Lipofectamine RNAiMAX reagent (Invitrogen, USA) according to the manufacturer's protocol. At $6 \mathrm{~h}$ after transfection, the medium was changed to RPMI 1640 containing 10\% FBS.

\section{Adenovirus transfection}

When the cell density reached 70-80\% confluence, H1975 cells were transfected with human CXCR4 adenoviral vectors (GenePharma, Shanghai, China) at a multiplicity of infection (MOI) of 100 for $6 \mathrm{~h}$ and were cultured for $24 \mathrm{~h}$ in RPMI 1640 containing 10\% FBS. The empty adenoviral vectors were used as negative control.

Cell proliferation assay

Cell proliferation was determined by the Cell Counting Kit-8 (CCK-8, Dojindo, Japan) assay. H1299 and H1975 cells were seeded into 96-well plates at a density of $5 \times 10^{3}$ cells per well. H1299 cells were transfected with siRNA against CXCR4 or a negative control siRNA, while H1975 cells were transfected with CXCR4 adenovirus or control vector. After 24,48 , or 72 h, $10 \mu \mathrm{L}$ of CCK-8 solution was added to each well, and 


\section{Cellular Physiology Cell Physiol Biochem 2018;50:1084-1099 \begin{tabular}{ll|l} 
and Biochemistry Published online: 24 October 2018 & $\begin{array}{l}\text { ○ 2018 The Author(s). Published by S. Karger AG, Basel } \\
\text { www.karger.com/cpb }\end{array}$ \\
\hline
\end{tabular}}

Liao et al.: CXCR4 and NSCLC- Induced Osteoclastogenesis

the incubation was continued for an additional $2 \mathrm{~h}$. The absorbance value (OD) was measured at $450 \mathrm{~nm}$ using a microplate reader (Varioskan Flash, Thermo Scientific, USA).

Invasion and migration assays

The invasion assay was performed using Transwell invasion chambers (Corning, NY, USA). H1299 and H1975 cells were plated in the top chamber in medium without serum and treated with $20 \mathrm{ng} / \mathrm{mL}$ of recombinant human CXCL12. Medium containing 10\% FBS was added to the bottom chamber. After 24 $\mathrm{h}$, the cells were fixed and stained with $0.1 \%$ crystal violet. Cells invading through the membrane were counted using a light microscope. For the migration assay, H1299 and H1975 cells were seeded in 12-well plates in medium containing $0.1 \%$ FBS. The wound was created by scratching with a $100-\mu$ l pipette tip, and the cells were treated with $20 \mathrm{ng} / \mathrm{mL}$ of recombinant human CXCL12 in 10\% FBS RPMI 1640 to stimulate cell migration. Images of wound healing were captured at 0,24 and $48 \mathrm{~h}$.

\section{ELISA detection}

The concentration of sVCAM1 was estimated using ELISA kits (BD Biosciences, USA). After treatment, the cell supernatants were isolated by centrifugation, and VCAM1 expression was detected according to the manufacturer's instructions. The absorbance was read at $450 \mathrm{~nm}$ using a microplate reader (Thermo Fisher, USA).

\section{Real-time PCR}

At the indicated time, total RNA was extracted from cells using TRIzol reagent (Invitrogen, USA). Firststrand complementary DNA was reversed from $1 \mathrm{mg}$ of total RNA using the Primer Script RT Reagent Kit (TaKaRa, Dalian, China). Quantitative real-time PCR was performed using a SYBR Green Premix Ex Taq Kit on a 7500 FAST LightCycler (ABI, USA) according to the manufacturer's protocol. The sequences of the primers used for real-time PCR are shown in Table 1. The relative expression of mRNA was normalized to GAPDH.

\section{Western blot}

The cells were lysed with RIPA buffer containing protease inhibitors (Beyotime, China). Proteins from the cell lysates were quantified using the BCA method, separated on an SDS-PAGE gel and transferred onto PVDF membranes. Next, the membranes were blocked and incubated with a specific primary antibody. The primary antibodies were used at the following dilutions: anti-CXCR4 (1:1000); anti-ADAM17 (1:1000); and anti-VCAM1 (1:500). After being washed, the membranes were incubated with an HRP-linked secondary antibody. The immunoreactive bands were detected using the Chemiluminescent HRP Substrate (Millipore, USA). GAPDH was re-probed to serve as a control.

\section{TRAP staining}

Tartrate-resistant acid phosphatasethe (TRAP) staining was performed with TRAP kits (Sigma, St. Louis, MO, USA). The 4- $\mu$ m-thick slices were incubated in the fixation solution for 30 seconds and transferred to the TRAP solution for $60 \mathrm{~min}$ at $37^{\circ} \mathrm{C}$. The nuclei were visualized with haematoxylin staining. For the in vitro osteoclastogenesis assay, RAW264.7 cells were plated in 24-well plates incubated with 10 $\mathrm{ng} / \mathrm{mL}$ RNAKL or combined with other treatments for 5 days. TRAP staining was applied according to the manufacturer's instructions. The stained sections and cells were viewed with an electron microscope (Olympus BX63, Tokyo, Japan). TRAP-positive multinucleated cells containing more than 3 nuclei were regarded as osteoclasts.

\section{Immunohistochemistry staining}

Immunohistochemistry was performed using the Polink-1 one-step polymer detection system (ZSGBBIO, China). Following deparaffinisation and rehydration, the sections were microwaved in $0.01 \mathrm{~mol} / \mathrm{L}$ sodium citrate $\left(80{ }^{\circ} \mathrm{C}\right.$ for $50 \mathrm{~min}$, followed by $40{ }^{\circ} \mathrm{C}$ for $10 \mathrm{~min}$ ) and incubated with anti- $\alpha$-SMA antibody 
primary antibodies (CXCR4, 1:50; VCAM1, 1:100; ADAM17, 1:50) overnight at $4{ }^{\circ} \mathrm{C}$. After incubation with a one-step polymer secondary antibody at room temperature for $50 \mathrm{~min}$, the signal was visualized using diaminobenzidine (DAB). Images were captured using an Olympus microscope (Tokyo, Japan).

\section{Statistics}

The data are presented as the mean \pm SEM. The Kaplan-Meier method and log-rank test were applied for survival time. The student's t-test or Mann-Whitney U test was used for comparison between two groups. Statistical significance was determined by one-way ANOVA among three group' comparisons, using SPSS 16.0 software (SPSS, Chicago, IL, USA); $p<0.05$ was considered significant.

\section{Results}

CXCR4 is related to osteoclastogenesis in human metastatic bone tissue in NSCLC

To investigate the role of CXCR4 expressed by NSCLC cell lines in the development of bone metastasis, we first detected CXCR4 expression in NSCLC human tissues. In the bone destruction area, CXCR4 is aberrantly expressed (Fig. 1A). Further, the osteoclastogenesis response in the bone destruction area on the consecutive slices, as evaluated using TRAP staining, was related to CXCR4 expression, as shown in Fig. 1A. The number of TRAP-positive multinucleated cells was considerably higher on the surface with high CXCR4 expression (Fig. 1A). Among the NSCLC bone metastasis patients undergoing surgery, 32 were followed up. Kaplan-Meier survival analysis implied that patients with high expression of CXCR4 seemed to suffer lower overall survival time rate as shown in Fig. 1B, consistent with previous clinical reports [21, 22]. A log-rank test further confirmed that a significant difference between overall survival time.

Fig. 1. CXCR4 expression in human specimens of lung cancer-induced bone metastasis. A. CXCR4 highly expressed in human specimens is associated with bone structure destruction. Left panel shows CXCR4 expression (200x) in the tumour-bone interface. Right panel showed osteoclastogenesis detection through TRAP staining in the tumourbone interface on the consecutive slices. B. The survival time of patients after surgery in varying CXCR4 expression group (CXCR4-/+ versus CXCR4 ++/+++). Kaplan-Meier survival analysis of overall survival in all patients with NSCLC bone metastasis was generated according to CXCR4 protein expression. The expression of CXCR4 was determined by immunostaining in samples from 31 NSCLC patients. Patients were divided into high CXCR4 expression group (n $=23$ ) (score ++ and +++ ) and low CXCR4 expression group $(\mathrm{n}=8)$ (score+ and -). The overall survival was compared between low and high CXCR4 expression groups. The log-rank test was applied to calculate $\mathrm{p}$ value.

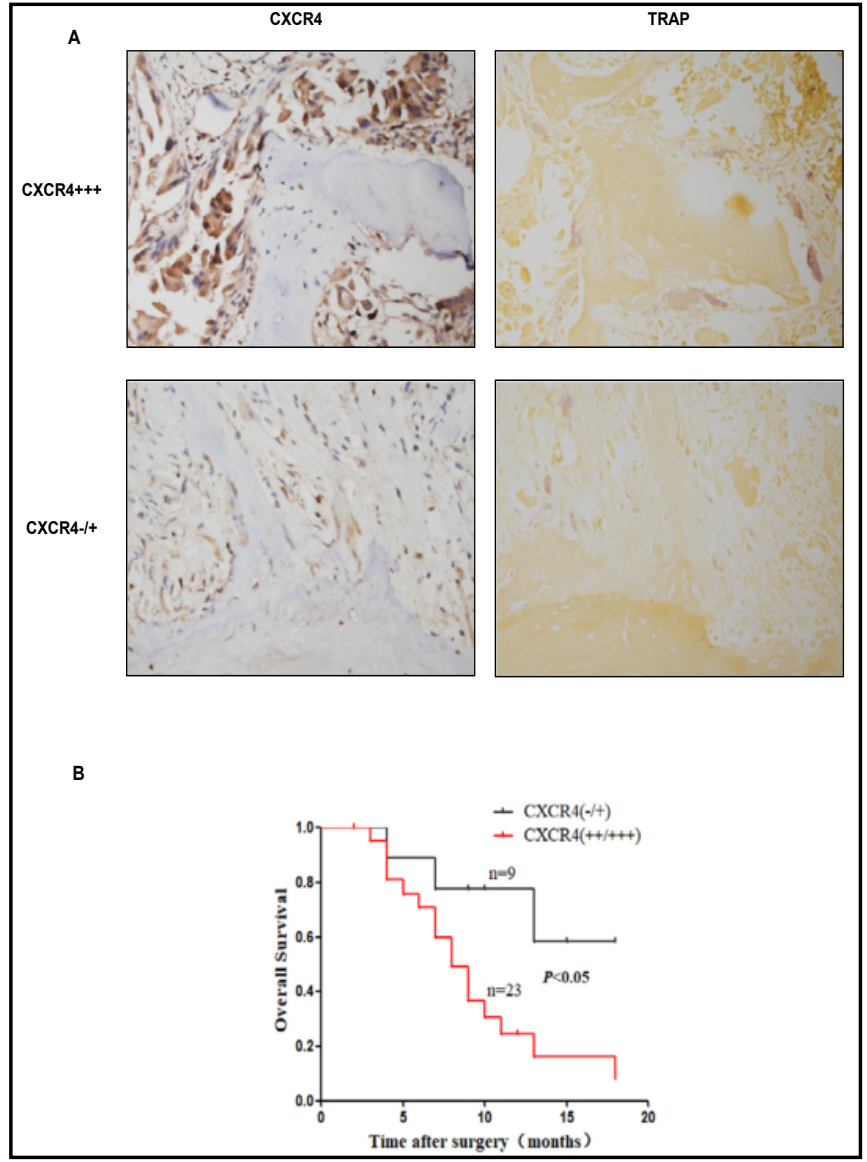


Fig. 2. Differential expression of CXCR4 mRNA in non-small cell lung carcinoma (NSCLC) cell lines. A. CXCR4 mRNA levels were measured via qRT-PCR in A549, H1975 and H1299 cell lines $\left(n=3,{ }^{*} p<0.05\right.$, compared with the A549 group). B. CXCR4 protein levels were measured via Western blot analysis in A549, H1975 and H1299 cell lines ( $n=3,{ }^{*} \mathrm{p}<0.05$, compared with the A549 group).

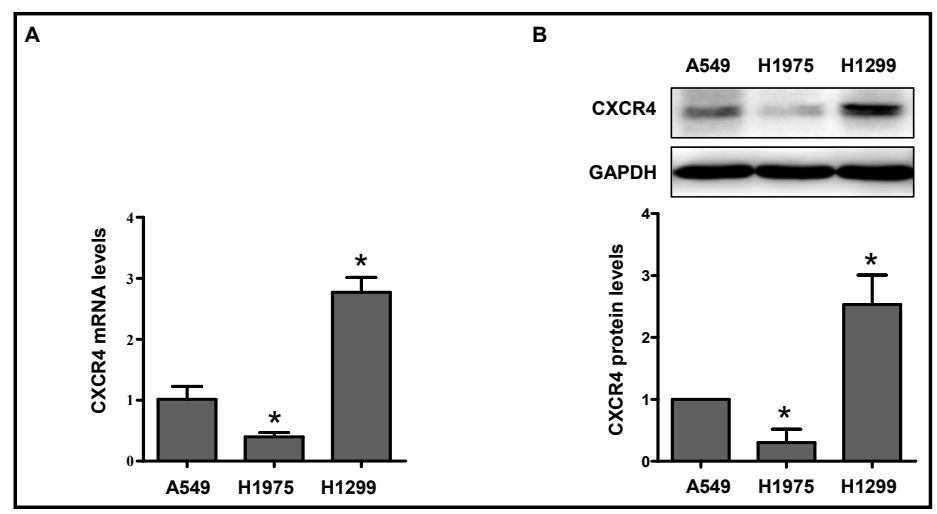

\section{CXCR4 knockdown inhibits NSCLC invasion, migration and proliferation in vitro}

To investigate the role of NSCLC CXCR4 in the development of bone metastasis, we first screened CXCR4 expression in several NSCLC cell lines, including A549, H1975 and H1299. Among them, the evaluation of both mRNA levels and protein expression showed that the CXCR4 expression was the highest in the H1299 cell line, while it was the lowest in the H1975 cells (Fig. 2A). Based on this result, we further evaluated CXCR4 in these cell lines to determine its effects on bone metastasis.

To determine the role of CXCR4 in NSCLC potentiation, three siRNA vectors were constructed and transfected into H1299 cells, in which CXCR4 was demonstrated to be relatively highly expressed (Fig. 2B). As shown in Fig. 3A, two of the siRNA against CXCR4, namely, si-CXCR4a and si-CXCR4b, showed high knockdown efficiency, while si-CXCR4c exhibited minimal effects. Consistently, si-CXCR4a effectively downregulated the CXCR4 protein expression (Fig. 3B) and, thus, was chosen for further studies.

Given the pivotal role of migration and invasion in cancer bone metastasis, we further determined the effects of CXCR4 knockdown in H1299 cells on invasive ability. As shown in Fig. 3C\&D, the wounded-monolayer assay showed that at both $24 \mathrm{~h}$ and $48 \mathrm{~h}$, siRNA against CXCR4 remarkably suppressed H1299 cell migration. Meanwhile, H1299 cells were seeded in the upper Transwell chamber and stimulated with serum to migrate into the bottom chamber. The cells that migrated into the lower membrane were counted. The results showed that the number of migrated cells in the CXCR4-knockdown group was significantly reduced compared with that in either the scrambled siRNA group or the control group (Fig. 3E). Additionally, we measured H1299 cell proliferation upon stimulation with CXCL12. In the CXCR4-knockdown group, the absorbance values were decreased at both $48 \mathrm{~h}$ and $72 \mathrm{~h}$ under stimulation with CXCL12 compared with that in the scrambled group. Taken together, these results indicated that knocking down CXCR4 in NSCLC could block the migratory, invasive and proliferative ability that vitally contribute to bone metastasis.

\section{CXCR4 overexpression accelerates NSCLC invasion, migration and proliferation in vitro}

In H1975 cells with relatively low CXCR4 expression, we overexpressed CXCR4 via an adenovirus to determine its effects on bone metastasis. We first confirmed the efficiency of the adenovirus transfection into the H1975 cells, and the mRNA levels were markedly increased, as shown in Fig. 4A. Correspondingly, the protein levels were increased to 2.38fold compared with the negative control group (Fig. 4B). In the wound-healing experiment, the healing distance was substantially greater in the CXCR4-overexpression group compared with the control group (Fig. 4C\&D). Likewise, the Transwell invasion assay showed that the migrated cells in the CXCR4-overexpression group were markedly increased compared with those in the control group (Fig. 4E). Moreover, the H1975 cells transfected with the CXCR4carrying adenovirus exhibited a remarkable increase in proliferation ability at both $48 \mathrm{~h}$ and $72 \mathrm{~h}$ compared with the control group (Fig. 4F). Therefore, these data indicated that CXCR4 overexpression potentiates NSCLC growth and invasion. 
Fig. 3. Effects of CXCR4 downregulation on the proliferation, migration and invasive ability in H1299 cell lines. A. CXCR4 was knocked down via siRNA transfection. The mRNA levels were measured with qRTPCR $\left(n=3,{ }^{*} p<0.05\right.$, compared with the control group or siRNA negative control group; NS, not significant). B. The CXCR4 protein levels were significantly decreased in the group transfected with siRNA against CXCR4 compared with that in the negative control group (n=3, *p $<0.05$, compared with the siRNA negative control group). C. The migration ability of H1299 cells in the presence or absence of siRNA CXCR4 was evaluated via the woundhealing assay. Representative

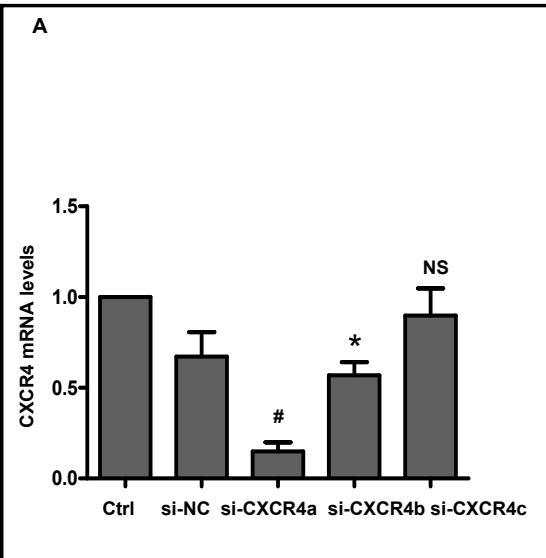

c

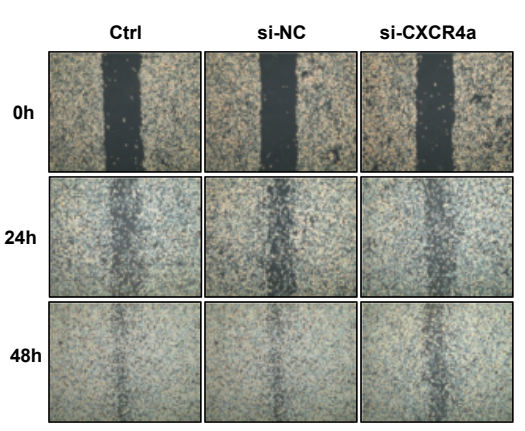

$E$
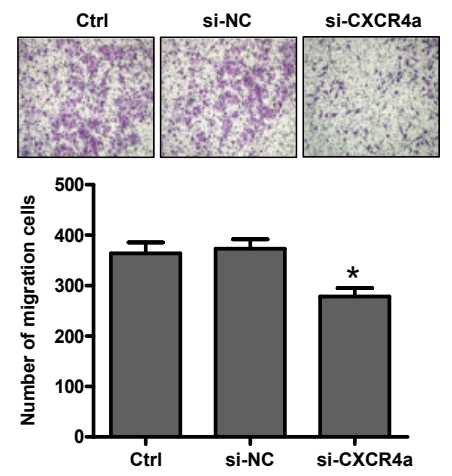
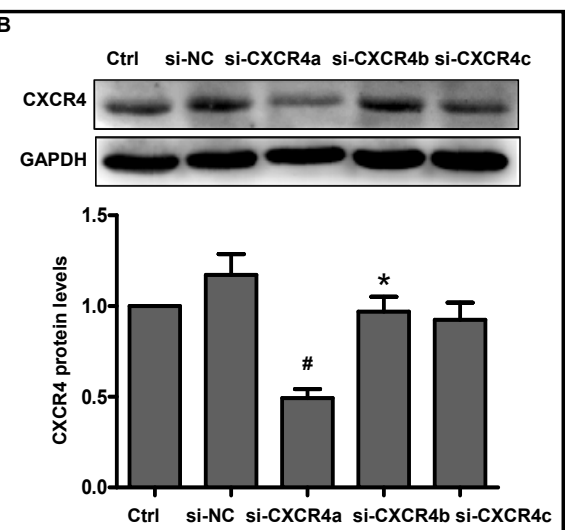

D

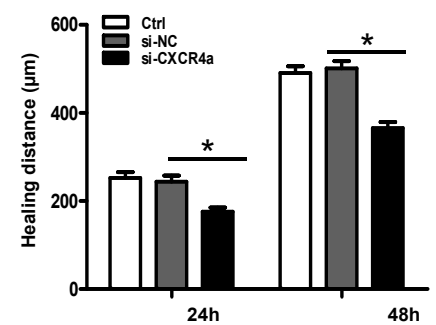

$\mathbf{F}$

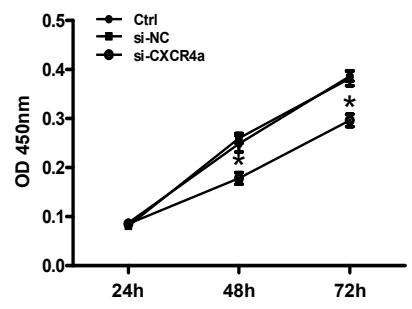

images were

recorded at 0, 24 and $48 \mathrm{~h}$ post-wound time points. D. Quantification of healing distance $\left(\mathrm{n}=3,{ }^{*} \mathrm{p}<0.05\right.$, compared with the control group or siRNA negative control group). E. Matrigel assay of H1299 cells transfected with siRNA against CXCR4 or control siRNA. Migratory cells were counted in 10 non-overlapping areas and quantified using a bar graph $\left(n=3,{ }^{*} \mathrm{p}<0.05\right.$, compared with the control group or siRNA negative control group). F. H1299 cell proliferation was measured using a CCK-8 kit. The OD values were measured at 24,48 and $72 \mathrm{~h}$ in the control group and CXCR4-knockdown group $\left(\mathrm{n}=5,{ }^{*} \mathrm{p}<0.05\right.$, compared with the siRNA negative control group).

\section{Role of CXCR4 in lung cancer-induced osteoclast differentiation and function}

The receptor activator of nuclear factor- $\kappa \mathrm{B}$ ligand (RANKL) could induce the formation of TRAP-positive multinucleated osteoclasts. Conditioned medium harvested from H1299 cells transfected with the negative control siRNA significantly promoted the differentiation of osteoclasts, as shown in Fig. 5A\&B. Conditioned medium from CXCR4-knockdown cells 
Fig. 4. Effects of CXCR4 overexpression on the proliferation, migration and invasive ability in H1975 cell lines. A. CXCR4 was overexpressed in H1975 cells via an adenovirus carrying CXCR4. The mRNA levels were measured using qRT-PCR $\quad\left(n=3,{ }^{*} \mathrm{p}<0.01\right.$ compared with negative control adenovirus transfection group). B. The CXCR4 protein levels were measured with Western blot in H1975 cells transfected with a CXCR4-carrying adenovirus $\quad(n=3$, ${ }^{\#} \mathrm{p}<0.01$ compared with the negative control adenovirus transfection group). C. The migration ability of H1975 cells in the presence or absence of the CXCR4 adenovirus were measured with the wound-healing assay. Representative images were recorded at 0,24 and $48 \mathrm{~h}$ postwound time points. D. Quantification of healing distance $(n=3$, *p $<0.05, \quad$ compared with the adenovirus negative control group). E. Matrigel invasive assay of H1975 transfected with the CXCR4 adenovirus or control vector. Migratory cells were counted in 10 non-overlapping areas and quantified as shown in the bar graph (each group $n=3$, with 10 visions, ${ }^{*} \mathrm{p}<0.05$, compared with the negative control adenovirus transfection group). F. H1975 cell proliferation was measured using a CCK-8 kit. The OD values were measured at 24, 48 and $72 \mathrm{~h}$ in the control group and the CXCR4 adenovirus transfection group $\left(\mathrm{n}=3,{ }^{*} \mathrm{p}<0.01\right.$ compared with the negative control adenovirus transfection group).

showed a decrease in TRAP-positive osteoclasts compared with that from the negative control group (Fig. 5A). Further, we measured the mRNA levels of TRAP and cathepsin K, which are markers of osteoclasts. Both TRAP and cathepsin K mRNA levels were increased in the presence of conditioned medium upon stimulation with RANKL (Fig. 5C). However, the increase in the TRAP and cathepsin K mRNA were blunted in the presence of the conditioned medium from the CXCR4-knockdown NSCLC cells (Fig. 5C). 
Fig. 5. Effects of CXCR4 manipulation in NSCLC on osteoclast progenitor cell differentiation. A. TRAP staining was performed to evaluate the formation of multinucleated cells. RAW264.7 cells were treated with $10 \mathrm{ng} / \mathrm{mL}$ RNAKL with or without H1299 culture medium (CM) harvested from the CXCR4knockdown group or the control transfection group. B. Quantification of TRAP-positive cells $\left(n=3,{ }^{*} p<0.05\right.$ compared with the negative control siRNA group). C. The mRNA levels of the osteoclast differentiation marker genes cathepsin $\mathrm{K}$ and TRAP in treated RAW264.7 cells were measured via RT-PCR at $48 \mathrm{~h}$ after treatment $\left(n=3,{ }^{*} p<0.05\right.$ compared with the negative control siRNA group). D. TRAP staining of osteoclast progenitor cells when CXCR4 was overexpressed. RAW264.7 cells were treated with $10 \mathrm{ng} / \mathrm{mL}$ RNAKL with or without H1975 CM harvested from the CXCR4-overexpression group compared with the adenovirus or control group. E. Quantification of TRAP-positive cells upon stimulation with cell culture media from CXCR4-overexpressing H1975 ( $\mathrm{n}=3,{ }^{*} \mathrm{p}<0.05$ compared with the adenovirus negative control group). F. mRNA levels of the osteoclast differentiation marker genes cathepsin $\mathrm{K}$ and TRAP in treated RAW264.7 cells were measured via RT-PCR at 48 $\mathrm{h}$ after treatment $\left(\mathrm{n}=3,{ }^{*} \mathrm{p}<0.01\right.$ compared with the negative control adenovirus transfection group). G. TRAP staining was performed to detect RAW264.7 cell differentiation following treatment with $10 \mathrm{ng} / \mathrm{mL}$ RNAKL and culture medium from CXCR4-overexpressing H1975 cells in the presence or absence of 10 $\mathrm{ng} / \mathrm{ml}$ AMD3100, as indicated. $\mathrm{H}$. Quantification of TRAP-positive cells upon stimulation with culture media from CXCR4-overexpressing H1975 cells in the presence or absence of AMD3100 ( $\left.n=3,{ }^{*} p<0.05\right)$. I. mRNA levels of osteoclast differentiation marker genes cathepsin K and TRAP in treated RAW264.7 cells were measured via RT-PCR at $48 \mathrm{~h}$ in the indicated groups $\left(\mathrm{n}=3,{ }^{*} \mathrm{p}<0.05\right)$.

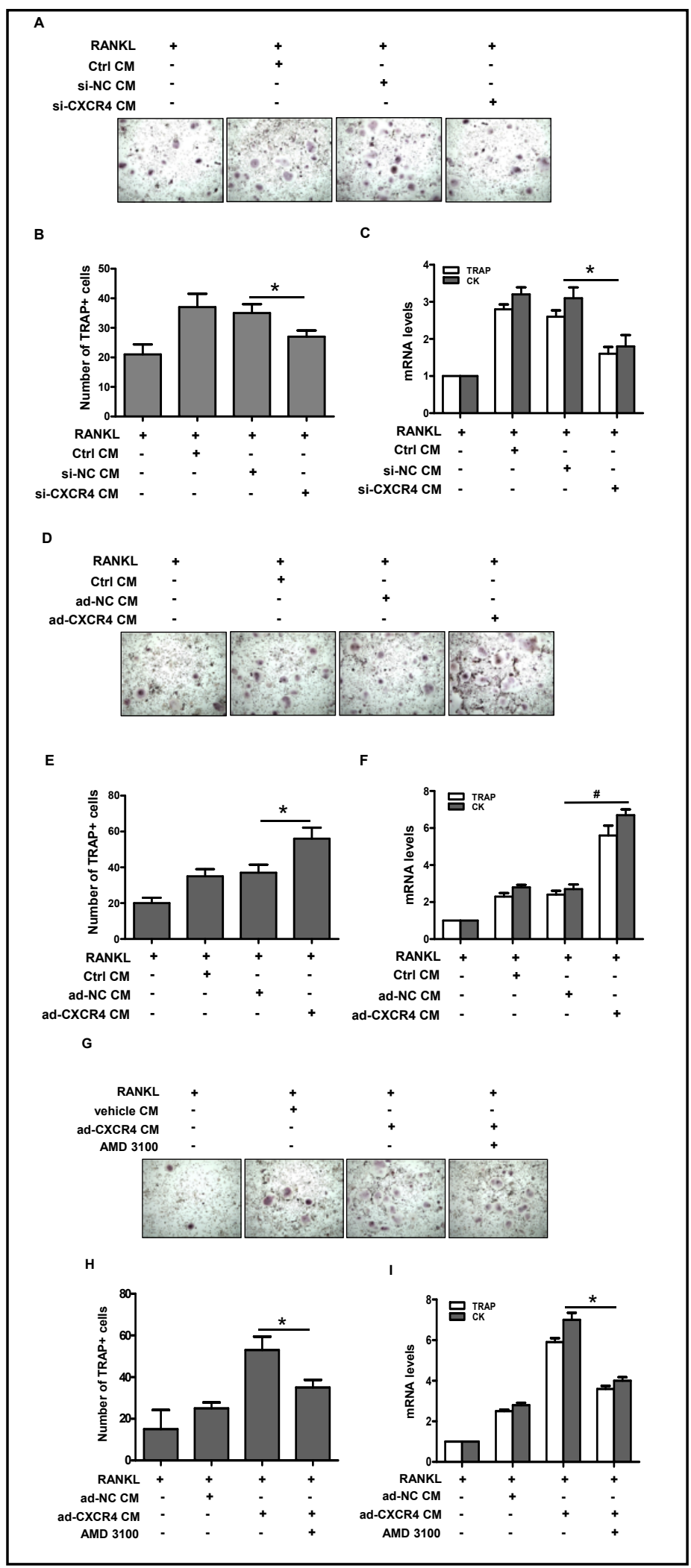

G 
On the other hand, we investigated the effects of conditioned medium from the CXCR4overexpressing H1975 cells on osteoclast differentiation. The conditioned medium from the CXCR4-overexpressing NSCLC cells dramatically stimulated osteoclast differentiation (Fig. 5D\&E). Similarly, the mRNA levels of TRAP and cathepsin K were upregulated in the group treated with the conditioned medium from the CXCR4-overexpressing NSCLC cells compared with that in the vector group (Fig. 5F).

AMD3100, a well-recognized CXCR4 antagonist, was further employed to determine the effects of CXCR4 inhibition on osteoclast differentiation. The conditioned medium harvested from the CXCR4-overexpressing NSCLC cells with the addition of AMD3100 showed less multinucleated osteoclasts stained with TRAP (Fig. 5G\&H). Likewise, the mRNA levels of TRAP and cathepsin $\mathrm{K}$ in the RAW264.7 cells were decreased upon stimulation with conditioned medium from the CXCR4-overexpression group in the presence of AMD3100 compared with that in the corresponding control group (Fig. 5I).

Fig. 6. VCMA1 mediated C X C R 4 - i n d u c e d osteoclastogenesis activity. A. The VCAM1 mRNA levels were measured with qRT-PCR in A549, H1975 and H1299 cell lines $\left(\mathrm{n}=3,{ }^{*} \mathrm{p}<0.05\right.$, compared with the A549 group). B. VCAM1 protein levels were determined after CXCR4 overexpression via adenovirus in $\mathrm{H} 1975$ cells $(\mathrm{n}=3, \quad * \mathrm{p}<0.05$ compared with the adenovirus negative control group). C. VCAM1 protein levels were measured when CXCR4 was knocked down via siRNA in H1299 cells $(n=3, \quad * p<0.05$ compared with the siRNA negative control group). D. The amount of soluble VCAM1 (sVCAM1) in the H1975 cell supernatants was detected using ELISA in the presence or absence of AMD3100 $\left(n=3, \quad{ }^{*} p<0.05\right) . \quad$ E. The mRNA levels of VCAM1 in H1299 cells overexpressing CXCR4 in the presence or absence of AMD3100, as indicated in the bar graph $(n=3, * p<0.05) . \quad F$. VCAM1-rich conditioned medium coating promoted the differentiation and osteoclastogenesis of RAW264.7. G. VCAM1 antibody supplementation blocked the VCAM1-mediated osteoclastogenesis of RAW264.7.

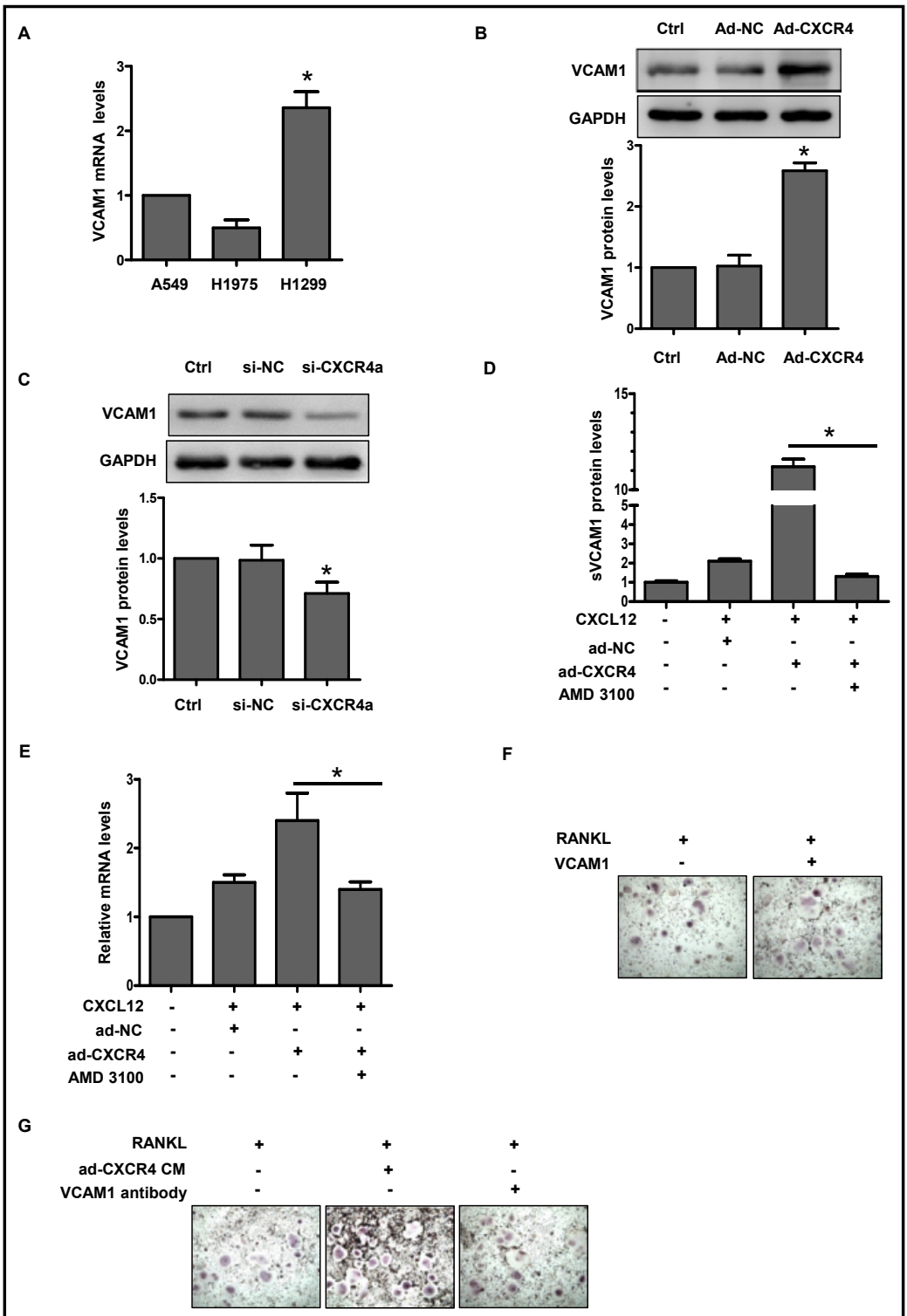

KARGER 
Fig. 7. ADAM17 inhibition reduced soluble VCAM1 in NSCLC conditioned medium. A. ADAM17 mRNA levels were measured via qRT-PCR in A549, H1975 and H1299 cell lines $(n=3$, ${ }^{*} \mathrm{p}<0.05$, compared with the A549 group). B. ADAM17 protein levels were measured via Western blot analysis in A549, H1975 and H1299 cell lines. C. ADAM17 expression was modulated by CXCR4 overexpression, as detected by Western blot analysis $(\mathrm{n}=3$, $\left.{ }^{*} \mathrm{p}<0.05\right)$. D. Protein levels of ADAM17 in the presence of TAPI2. E. TRAP staining of osteoclast progenitor cells cultured with conditioned medium from NSCLC cells overexpressing CXCR4 in the presence of TAPI2. F. Quantification of Fig. 7E $\left(n=3,{ }^{*} p<0.05\right.$, $\left.{ }^{\#} \mathrm{p}<0.01\right)$. G. mRNA levels of osteoclast differentiation marker genes cathepsin $\mathrm{K}$ and TRAP in the groups indicated in the bar graph $(n=3, * p<0.05$, $\left.{ }^{\#} \mathrm{p}<0.01\right)$. H. ADAM17 inhibition with TAPI-2 reduced the expression of VCAM1 in NSCLC determined by qRT-PCR $(n=3$, ${ }^{\#} \mathrm{p}<0.01$ ). I. ADAM17 inhibition reduced soluble VCAM1 in the conditioned medium $(\mathrm{n}=3, \quad$ " $\mathrm{p}<0.01) . \quad$ J. CXCR4, VCAM1, and
A

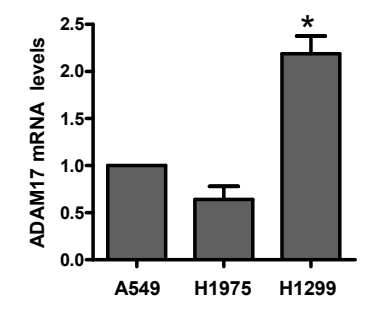

c

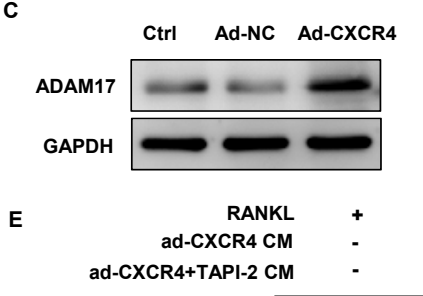

B
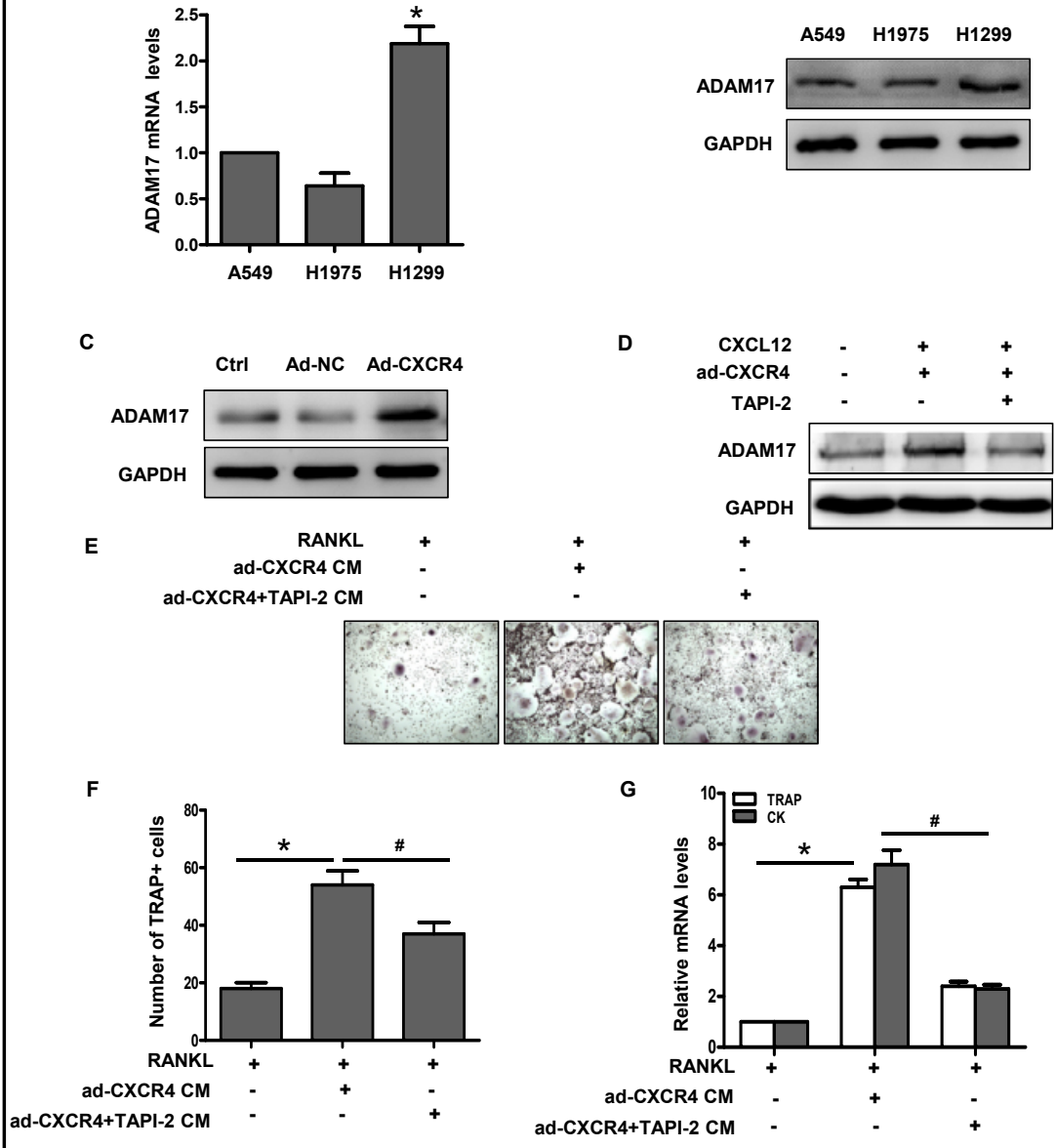

H
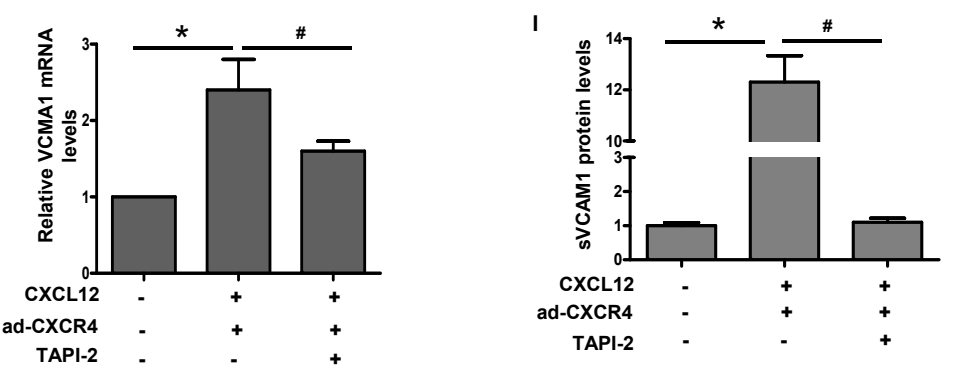

J
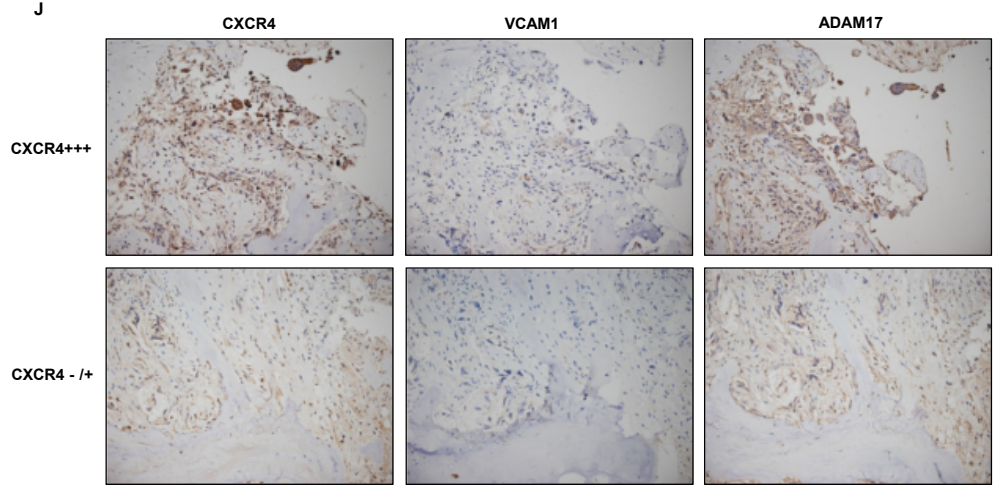

ADAM17 expression in human specimens on consecutive slices with immunohistochemistry. 
VCAM1 is involved in the CXCR4-positive-NSCLC-induced osteoclast activation

We first measured VCAM1 expression in different NSCLC cell lines. Consistent with CXCR4 expression, the mRNA levels of VCAM1 in the H1299 cells are higher than those in the H1975 cell lines, as shown in Fig. 6A. Aberrant CXCR4 overexpression induced significant VCAM1 expression in H1975 cells (Fig. 6B), while CXCR4 downregulation decreased VCAM1 expression in H1299 cells (Fig. 6C). In the CXCR4-overexpression group, sVCAM1 levels in the culture medium were increased compared with those in the control group (Fig. 6D). The CXCR4 inhibitor AMD3100 decreased the secretion of sVCAM1 to the medium, which was enhanced by CXCR4 overexpression in the presence of CXCL12 (Fig. 6D). In the NSCLC cells, the increase in the VCAM1 mRNA induced by CXCR 4 overexpression was blunted by AMD3100 (Fig. 6D\&E). Moreover, VCAM1 might interact with its receptor $\alpha 4 \beta 1$ on preosteoclasts to promote osteoclastogenesis [17]. We mimicked that scenario by precoating the wells with VCAM1-rich conditioned medium from CXCR4-overexpressing NSCLC cells before seeding RAW264.7 cells. The coating with the VCAM1-rich conditioned medium resulted in earlier multi-nucleation and more TRAP+ osteoclasts (Fig. 6F). On the other hand, supplementation with a VCAM1 antibody blocked the maturation of the RAW264.7 cells seeded in the VCAM1-coated conditioned medium and reduced the multinucleated TRAP+ osteoclasts (Fig. 6G). Taken together, these results indicated that the sVCAM1 secreted from the CXCR4overexpressing NSCLC cells may recruit and arrest more osteoclast progenitors to promote osteoclastogenesis.

ADAM17 might mediate VCAM1 secretion in CXCR4-overexpressing NSCLC tumour cells and participate in CXCR4-accelerated osteoclastogenesis

Previously, it has been reported that ADAM17 is involved in VCAM1 cleavage and facilitates the secretion of sVCAM1 [18]. We determined whether ADAM17 acts as a downstream regulator of CXCR4. ADAM17 was detected in different NSCLC cell lines with varying CXCR4 expression. We observed that ADAM17 has a similar expression profile as CXCR4 (Fig. 7A\&B). CXCR4 overexpression led to the upregulation of ADAM17 (Fig. 7C). In the presence of the ADAM17 inhibitor TAPI-2, the ADAM17 expression induced by CXCR4 was decreased compared with that in the control group (Fig. 7D). Further, the suppression of ADAM17 decreased the number of TRAP+ osteoclasts (Fig. 7E \&F). The osteoclast marker genes TRAP and CK were also inhibited in the presence of TAPI-2 (Fig. 7G).

Meanwhile, CXCR4 overexpression via adenovirus upregulated the VCAM1 expression in NSCLC cells, as described above (Fig. 6B). We further observed that inhibition of ADAM17 via TAPI- 2 attenuated the elevation of VCAM1 induced by CXCR 4 at both mRNA levels and the soluble form in NSCLC cells (Fig. 7H\&I). Likewise, the sVCAM1 in the conditioned medium was significantly lower in the presence of TAPI-2 (Fig. 7H). Therefore, we could infer that ADAM17 functions as a downstream moderator during CXCR4-accelerated osteoclastogenesis and that ADAM17 plays a vital role in the release of sVCAM1 into the cell medium, which acts on osteoclast progenitors and, eventually, leads to activation of osteoclastogenesis.

We recapitulated our observation in the human samples. Consecutive slices immunostaining showed that higher CXCR4 expression in the NSCLC metastatic bone tissues correlate with aberrant expression of VCAM1, and ADAM17 compared with CXCR4 low expression group (Fig. 7J). This observation in human samples further supported the mechanisms that ADAM17 might participate in the regulation of VCAM1 by CXCR4 during NSCLC metastasis to bone.

\section{Discussion}

NSCLC remains a life-threatening disease, and its metastasis to bone accelerates disease progression. To develop targeted therapeutic modalities, it is important to delineate the mechanism underlying the bone metastasis of NSCLC. In this report, we confirmed the differential expression of CXCR4 in different NSCLC cell lines and the distribution of CXCR4 
in human tissue samples of bone metastasis sites. CXR4 expression is related to enhanced osteoclastogenesis. In addition, we further manipulated CXCR4 expression in NSCLC and revealed that CXCR4 overexpression results in enhanced NSCLC proliferation and invasive capability. The conditioned medium from CXCR4-overexpressing NSCLC cells promoted osteoclastogenesis. We further demonstrated that sVCAM1 cleaved by ADAM17 acts as downstream modulator during CXCR4-induced osteoclastogenesis in NSCLC.

Tumour cells disseminate from the tumour mass and metastasize to distant tissues. The bone has physical properties that sustain tumour cell proliferation and provide a large surface, a hypoxic environment and growth factors. Approximately $36 \%$ of patients with multiple skeleton-favouring tumours present with bone lesions at autopsy [23]. The median survival time from the diagnosis of advanced lung cancer is usually counted in months [23]. Despite the short survival time, SREs developed at a high rate. Moreover, some NSCLC patients are diagnosed because of pain and SREs. The progression to SREs leads to a reduction in quality of life and a poor prognosis. Therefore, studying the mechanisms accounting for the bone metastasis of NSCLC is of great importance to improve the outcome of lung cancer patients.

Increasing studies have shown that CXCR4 is significantly elevated in a wide range of tumours, such as lung cancer and gastric cancer [24, 25]. Not limited to the in-site tumours, aberrant CXCR4 overexpression was detected in circulating tumour cells and tumour metastasis target organs, including lung, brain, bone and liver. Clinically, a growing body of evidence has shown that CXCR4 predicts poor prognosis of patients with lung cancer [26]. A meta-analysis indicated that CXCR4 expression is associated with an increased risk and worse survival in NSCLC patients [27].

CXCR4 is a chemokine receptor expressed by NSCLC that can bind to factors produced by the bone and bone marrow, such as stromal derived factor-1 (SDF1). Tumour cells achieve local reabsorption through the osteoclastogenesis process. During osteoclastogenesis, tumour cells activate osteoclast precursor cells of the monocyte/macrophage cell line and stimulate their fusion and formation of mature osteoclasts. Among multiple osteoclastogenesis signalling pathways, the RNAKL/RANK/osteoprotegerin system is the most recognized axis. In this study, we observed that CXCR4 expression was considerably different among various NSCLC cell lines and human samples. The differences were tightly related to clinical outcomes. Our finding was consistent with other clinical investigations [26, $28,29]$. Liu et al. reported that NSCLC with CXCR4 expression has a higher odds ratio with late-stage lung cancer, lymph node involvement and bone metastasis [26]. We found that CXCR4 exhibited a significant positive correlation with overall survival time. Furthermore, we demonstrated that the poor outcome could be explained by the potentiation of NSCLC through the aberrant expression of CXCR4. CXCR4-positive tumours exhibited accelerated proliferation and invasion ability. Hence, CXCR4 might serve as a prognostic factor for lung cancer patient survival and might be an emerging therapeutic target.

VCAM1 is a well-known cell surface immunoglobulin molecule. It has been reported to be constitutively expressed on several different cells, including endothelial cells, stromal cells and many tumour cells. VCAM1 mediates cell adhesion physically and, thus, appears to be of special interest for its pivotal role in the process of tumour invasion and metastatic spread. According to previously published literature, sVCAM1 levels are elevated in patients with lung cancer [30,31], breast cancer and even colorectal carcinomas compared with control subjects [32-34]. In this study, we confirmed that in human metastatic samples from bone, VCAM1 was upregulated on the tumour-bone surface in the bone destruction area. Consistently, we further discovered that sVCAM1 was increased in the conditioned medium from the CXCR4-overexpressing NSCLC cells and that it promoted osteoclastogenesis through interaction with osteoclast progenitors. This result supported an observation in breast-cancer-induced metastasis. In breast cancer bone metastasis, VCAM1 was indicated to promote osteolytic expansion through binding to its cognate $\alpha 4 \beta 1$ receptor in osteoclast progenitors [17]. Therefore, we speculated that the interaction between VCAM 1 and $\alpha 4 \beta 1$ might contribute to increased osteoclastogenesis in NSCLC. Moreover, increased expression of CXCR4 and VLA-4 integrin resulted in concentration-dependent chemotaxis to their

\section{KARGER}


ligands, CXCL12 and VCAM-1 in T cells [35]. The cross talk between CXCL12/CXCR4 and VLA4/VCAM-1 was further validated in the bone marrow [36]. Previously reports also showed that in astrocytes CXCL12 stimulation could induce the NF $\kappa B$ activation and TNF $\alpha$ release [37], concomitantly the transcription regulation between NF $\kappa B$ and VCAM1 has been well established. Hence, we reasoned that NF $\mathrm{KB}$ might be one of the possible mechanisms CXCR4 regulates VCAM1. However, further investigation is still needed.

ADAM17 belongs to the disintegrin and metalloprotease gene family [38], and it has been reported to be upregulated in NSCLC and other tumour entities. Aberrant ADAM17 expression was associated with a poor survival time [39]. ADAM17 can be activated by radiotherapy in NSCLC, which results in shedding of multiple survival factors and growth factor pathway activation [40]. A previous study showed that ADAM17 downregulation suppresses NSCLC migration and invasion [38]. Our results extend this observation; we found that ADAM17 was regulated by CXCR4. Meanwhile, our results indicated that ADAM17 inhibition inactivated osteoclastogenesis. In murine endothelial cells, ADAM17 was reported to serve as the protease responsible for shedding of endogenous VCAM1 and sVCAM1 release [18]. Therefore, we reasoned that VCAM1 might contribute to ADAM17-mediated osteoclastogenesis. ADAM17 inhibition decreased NSCLC VCAM1 expression and led to a reduction of sVCAM1 in the conditioned medium. In summary, we innovatively established a cross talk between the CXCL12/CXCR4 axis and the ADAM17/VCAM1 interaction.

The limitations of this study should be taken into consideration. First, our findings need to be further evaluated using in vivo animal models. In vivo tumour models could be more convincing to demonstrate the role of CXCR4 in tumorigenesis and bone metastasis. Second, we performed studies on human samples and follow-up studies in patients with NSCLC. However, a prospective cohort study might illustrate the predictive value of CXCR4 and sVCAM1 for prognosis. Finally, it would be valuable to determine the concrete function of VCAM1 during osteoclastogenesis.

\section{Conclusion}

We determined the important role of CXCR4 in NSCLC-induced bone metastasis. CXCR4 appears to potentiate the outgrowth of NSCLC, promote the invasion ability of NSCLC, and enhance osteoclastogenesis through sVCAM1. Further, we confirmed that ADAM17 is involved in sVCAM1 cleavage and participates in NSCLC-mediated osteoclastogenesis. The cross talk between CXCR4 signalling and the ADAM17 cleavage of VCAM1 were established in this study. These data may indicate the potential therapeutic value of the CXCR4-ADAM17VCAM1 axis.

\section{Acknowledgements}

The authors would like to thank Rong Xin for the technique assistance in the immunohistochemistry staining experiment. This project was funded by the National Natural Science Foundation of China (Grant number 81570800).

\section{Disclosure Statement}

The authors declare to have no competing interests. 


\section{Cellular Physiology Cell Physiol Biochem 2018;50:1084-1099 \begin{tabular}{l|l|l|l|l}
\hline DOI: 10.1159/000494533 & (c) 2018 The Author(s). Published by S. Karger AG, Basel \\
whw
\end{tabular} and Biochemistry Published online: 24 October 2018 wh.katger.com/cpb}

\section{References}

1 Mitsudomi T, Kosaka T, Endoh H, Horio Y, Hida T, Mori S, Hatooka S, Shinoda M, Takahashi T, Yatabe Y: Mutations of the epidermal growth factor receptor gene predict prolonged survival after gefitinib treatment in patients with non-small-cell lung cancer with postoperative recurrence. J Clin Oncol 2005;23:2513-2520.

- Wang T, Han S, Wu Z, Han Z, Yan W, Liu T, Wei H, Song D, Zhou W, Yang X, Xiao J: XCR1 promotes cell growth and migration and is correlated with bone metastasis in non-small cell lung cancer. Biochem Biophys Res Commun 2015;464:635-641.

-3 Yu D, Lei JQ, Guo SL, Yu Q: The CNPY2 enhances epithelial-mesenchymal transition via activating the AKT/ GSK3beta pathway in non-small cell lung cancer. Cell Biol Int 2018;10.1002/cbin.10961.

-4 Suva LJ, Washam C, Nicholas RW, Griffin RJ: Bone metastasis: mechanisms and therapeutic opportunities. Nat Rev Endocrinol 2011;7:208-218.

-5 Weidle UH, Birzele F, Kollmorgen G, Ruger R: Molecular Mechanisms of Bone Metastasis. Cancer Genomics Proteomics 2016;13:1-12.

6 Xu C, Zhao H, Chen H, Yao Q: CXCR4 in breast cancer: oncogenic role and therapeutic targeting. Drug Des Devel Ther 2015;9:4953-4964.

7 Kuroda K, Fukuda T, Krstic-Demonacos M, Demonacos C, Okumura K, Isogai H, Hayashi M, Saito K, Isogai E: miR-663a regulates growth of colon cancer cells, after administration of antimicrobial peptides, by targeting CXCR4-p21 pathway. BMC Cancer 2017;17:33.

-8 Zhao H, Guo L, Zhao H, Zhao J, Weng H, Zhao B: CXCR4 over-expression and survival in cancer: a system review and meta-analysis. Oncotarget 2015;6:5022-5040.

$\checkmark 9$ Hirbe AC, Morgan EA, Weilbaecher KN: The CXCR4/SDF-1 chemokine axis: a potential therapeutic target for bone metastases? Curr Pharm Des 2010;16:1284-1290.

10 Phillips RJ, Burdick MD, Lutz M, Belperio JA, Keane MP, Strieter RM: The stromal derived factor-1/CXCL12CXC chemokine receptor 4 biological axis in non-small cell lung cancer metastases. Am J Respir Crit Care Med 2003;167:1676-1686.

-11 Spano JP, Andre F, Morat L, Sabatier L, Besse B, Combadiere C, Deterre P, Martin A, Azorin J, Valeyre D, Khayat D, Le Chevalier T, Soria JC: Chemokine receptor CXCR4 and early-stage non-small cell lung cancer: pattern of expression and correlation with outcome. Ann Oncol 2004;15:613-617.

12 Zuo J, Wen M, Li S, Lv X, Wang L, Ai X, Lei M: Overexpression of CXCR4 promotes invasion and migration of non-small cell lung cancer via EGFR and MMP-9. Oncol Lett 2017;14:7513-7521.

13 Hu X, Mei S, Meng W, Xue S, Jiang L, Yang Y, Hui L, Chen Y, Guan MX: CXCR4-mediated signaling regulates autophagy and influences acute myeloid leukemia cell survival and drug resistance. Cancer Lett 2018;425:1-12.

14 Al Husaini H, Wheatley-Price P, Clemons M, Shepherd FA: Prevention and management of bone metastases in lung cancer: a review. J Thorac Oncol 2009;4:251-259.

-15 Luo T, Liu H, Feng W, Liu D, Du J, Sun J, Wang W, Han X, Guo J, Amizuka N, Li X, Li M: Adipocytes enhance expression of osteoclast adhesion-related molecules through the CXCL12/CXCR4 signalling pathway. Cell Prolif 2017;50.

-16 Osborn L, Hession C, Tizard R, Vassallo C, Luhowskyj S, Chi-Rosso G, Lobb R: Direct expression cloning of vascular cell adhesion molecule 1, a cytokine-induced endothelial protein that binds to lymphocytes. Cell 1989;59:1203-1211.

17 Lu X, Mu E, Wei Y, Riethdorf S, Yang Q, Yuan M, Yan J, Hua Y, Tiede BJ, Lu X, Haffty BG, Pantel K, Massague J, Kang Y: VCAM-1 promotes osteolytic expansion of indolent bone micrometastasis of breast cancer by engaging alpha4beta1-positive osteoclast progenitors. Cancer Cell 2011;20:701-714.

18 Garton KJ, Gough PJ, Philalay J, Wille PT, Blobel CP, Whitehead RH, Dempsey PJ, Raines EW: Stimulated shedding of vascular cell adhesion molecule 1 (VCAM-1) is mediated by tumor necrosis factor-alphaconverting enzyme (ADAM 17). J Biol Chem 2003;278:37459-37464.

19 Miller MA, Sullivan RJ, Lauffenburger DA: Molecular Pathways: Receptor Ectodomain Shedding in Treatment, Resistance, and Monitoring of Cancer. Clin Cancer Res 2017;23:623-629.

20 Mustafi R, Dougherty U, Mustafi D, Ayaloglu-Butun F, Fletcher M, Adhikari S, Sadiq F, Meckel K, Haider HI, Khalil A, Pekow J, Konda V, Joseph L, Hart J, Fichera A, Li YC, Bissonnette M: ADAM17 is a Tumor Promoter and Therapeutic Target in Western Diet-associated Colon Cancer. Clin Cancer Res 2017;23:549-561. 


\section{Cellular Physiology Cell Physiol Biochem 2018;50:1084-1099

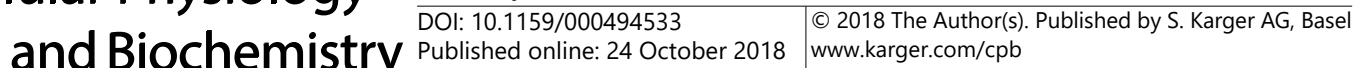 and Biochemistry Published online: 24 October 2018 ,}

-21 Al Zobair AA, Al Obeidy BF, Yang L, Yang C, Hui Y, Yu H, Zheng F, Yang G, Xie C, Zhou F, Zhou Y: Concomitant overexpression of EGFR and CXCR4 is associated with worse prognosis in a new molecular subtype of nonsmall cell lung cancer. Oncol Rep 2013;29:1524-1532.

22 Otsuka S, Klimowicz AC, Kopciuk K, Petrillo SK, Konno M, Hao D, Muzik H, Stolte E, Boland W, Morris D, Magliocco AM, Bebb DG: CXCR4 overexpression is associated with poor outcome in females diagnosed with stage IV non-small cell lung cancer. J Thorac Oncol 2011;6:1169-1178.

-23 Coleman RE: Clinical features of metastatic bone disease and risk of skeletal morbidity. Clin Cancer Res 2006;12:6243s-6249s.

-24 Wald O, Shapira OM, Izhar U: CXCR4/CXCL12 axis in non small cell lung cancer (NSCLC) pathologic roles and therapeutic potential. Theranostics 2013;3:26-33.

25 Iwasa S, Yanagawa T, Fan J, Katoh R: Expression of CXCR4 and its ligand SDF-1 in intestinal-type gastric cancer is associated with lymph node and liver metastasis. Anticancer Res 2009;29:4751-4758.

26 Liu K, Bao C, Yao N, Miao C, Varlotto J, Sun Q, Sun X: Expression of CXCR4 and non-small cell lung cancer prognosis: a meta-analysis. Int J Clin Exp Med 2015;8:7435-7445.

27 Zhang C, Li J, Han Y, Jiang J: A meta-analysis for CXCR4 as a prognostic marker and potential drug target in non-small cell lung cancer. Drug Des Devel Ther 2015;9:3267-3278.

-28 Katsura M, Shoji F, Okamoto T, Shimamatsu S, Hirai F, Toyokawa G, Morodomi Y, Tagawa T, Oda Y, Maehara Y: Correlation between CXCR4/CXCR7/CXCL12 chemokine axis expression and prognosis in lymph-nodepositive lung cancer patients. Cancer Sci 2018;109:154-165.

29 Wagner PL, Hyjek E, Vazquez MF, Meherally D, Liu YF, Chadwick PA, Rengifo T, Sica GL, Port JL, Lee PC, Paul S, Altorki NK, Saqi A: CXCL12 and CXCR4 in adenocarcinoma of the lung: association with metastasis and survival. J Thorac Cardiovasc Surg 2009;137:615-621.

-30 Staal-van den Brekel AJ, Thunnissen FB, Buurman WA, Wouters EF: Expression of E-selectin, intercellular adhesion molecule (ICAM)-1 and vascular cell adhesion molecule (VCAM)-1 in non-small-cell lung carcinoma. Virchows Arch 1996;428:21-27.

-31 Tas F, Karabulut S, Bilgin E, Duranyildiz D: Serum levels of vascular cell adhesion molecule-1 (VCAM-1) may have diagnostic, predictive, and prognostic roles in patients with lung cancer treated with platinum-based chemotherapy. Tumour Biol 2014;35:7871-7875.

-32 Silva HC, Garcao F, Coutinho EC, De Oliveira CF, Regateiro FJ: Soluble VCAM-1 and E-selectin in breast cancer: relationship with staging and with the detection of circulating cancer cells. Neoplasma 2006;53:538-543.

-33 Okugawa Y, Miki C, Toiyama Y, Koike Y, Inoue Y, Kusunoki M: Serum level of soluble vascular cell adhesion molecule 1 is a valuable prognostic marker in colorectal carcinoma. Dis Colon Rectum 2009;52:1330-1336.

34 Mantur M, Snarska J, Koper O, Dzieciol J, Plonski A, Lemancewicz D: Serum sICAM, sVCAM and sE-selectin levels in colorectal cancer patients. Folia Histochem Cytobiol 2009;47:621-625.

-35 Bryant J, Ahern DJ, Brennan FM: CXCR4 and vascular cell adhesion molecule 1 are key chemokine/adhesion receptors in the migration of cytokine-activated T cells. Arthritis Rheum 2012;64:2137-2146.

-36 Petty JM, Lenox CC, Weiss DJ, Poynter ME, Suratt BT: Crosstalk between CXCR4/stromal derived factor-1 and VLA-4/VCAM-1 pathways regulates neutrophil retention in the bone marrow. J Immunol 2009;182:604-612.

-37 Han Y, He T, Huang DR, Pardo CA, Ransohoff RM: TNF-alpha mediates SDF-1 alpha-induced NF-kappa B activation and cytotoxic effects in primary astrocytes. J Clin Invest 2001;108:425-435.

38 Lv X, Li Y, Qian M, Ma C, Jing H, Wen Z, Qian D: ADAM17 silencing suppresses the migration and invasion of non-small cell lung cancer. Mol Med Rep 2014;9:1935-1940.

39 Ni SS, Zhang J, Zhao WL, Dong XC, Wang JL: ADAM17 is overexpressed in non-small cell lung cancer and its expression correlates with poor patient survival. Tumour Biol 2013;34:1813-1818.

40 Sharma A, Bender S, Zimmermann M, Riesterer O, Broggini-Tenzer A, Pruschy MN: Secretome Signature Identifies ADAM17 as Novel Target for Radiosensitization of Non-Small Cell Lung Cancer. Clin Cancer Res 2016;22:4428-4439. 